\title{
Multiuser Detection in Fast-Fading Multipath Environments
}

\author{
Akbar M. Sayeed, Member, IEEE, Andrew Sendonaris, Student Member, IEEE, \\ and Behnaam Aazhang, Senior Member, IEEE
}

\begin{abstract}
We propose a new framework for multiuser detection in fast-fading channels that are encountered in many mobile communication scenarios. Existing multiuser RAKE receivers, developed to combat multipath fading and multiuser interference in slow fading, suffer substantial degradation in performance under fast fading due to errors in channel state estimation. The detectors proposed in this paper employ a novel receiver structure based on time-frequency (TF) processing that is dictated by a canonical representation of the wide-sense stationary uncorrelated scatterer (WSSUS) channel model. The workhorse of the framework is a TF generalization of the RAKE receiver that exploits joint multipath-Doppler diversity. Analytical and simulated results based on realistic fast-fading assumptions demonstrate that the proposed multiuser detectors promise substantially improved performance compared to existing systems due to the inherently higher level of diversity afforded by multipath-Doppler processing.
\end{abstract}

Index Terms - Doppler, fast fading, multipath, multiuser detection, RAKE receiver.

\section{INTRODUCTION}

C ODE division multiple access (CDMA) has emerged as one of the most promising systems for multiuser wireless communication. The need for accommodating the growing number of users and for communication in diverse environments has posed unique technological challenges in system design. Two of the most significant factors limiting the performance of existing mobile wireless CDMA systems are multipath fading and multiple access interference. Multipath fading is due to the channel dynamics produced by the multiple mobile scatterers encountered in transmission. Multiple access interference, on the other hand, is caused by the multiple users simultaneously using the channel. The RAKE receiver structure is used in practice to combat fading [1], [2] and various multiuser detection schemes have been proposed [3] to overcome multiple access interference. Recently, multiuser RAKE receivers have been proposed to combat multiple access interference in fading channels [4], [5]. Such schemes,

Manuscript received September 1997; revised March 1998. This work was supported in part by the Texas Advanced Technology Program under Grants 003604-049 and 003604-044 and by the NSF under Grant NCR-9506681. This paper was presented in part at the 31st Asilomar Conference on Signals, Systems, and Computers, Pacific Grove, CA, November, 1997.

A. M. Sayeed is with the Department of Electrical and Computer Engineering, University of Wisconsin, Madison, WI 53706 USA (e-mail: akbar@engr.wisc.edu.).

A. Sendonaris and B. Aazhang are with the Department of Electrical and Computer Engineering, Rice University, Houston, TX 77005 USA (e-mail: sendos@rice.edu.)

Publisher Item Identifier S 0733-8716(98)08644-2. however, are applicable only in slow fading scenarios in which the channel characteristics change slowly over time.

Fast fading encountered in many mobile communication scenarios significantly degrades the performance of the RAKE receiver due to less reliable channel estimation [6]-[8]. In fact, existing systems exhibit a limiting bit-error probability floor that cannot be improved by increasing the transmitted power [6], [8]. Recently, a single-user spread-spectrum communication scheme has been proposed for fast-fading channels that exploits temporal channel variations to provide another means for diversity-Doppler diversity - to counter such degradation in performance [9]-[11]. The methodology uses joint timefrequency (TF) processing which is a powerful approach to time-varying signal processing [12]. At the heart of the approach is a fundamental time-frequency-based channel decomposition derived from a sampling of the wide-sense stationary uncorrelated scatterer (WSSUS) model. The channel representation naturally leads to a time-frequency generalization of the RAKE receiver that exploits joint multipath-Doppler diversity. The TF RAKE receiver can deliver substantially improved performance compared to existing systems by achieving an inherently higher level of diversity [9]-[11].

In this paper, we leverage the TF formulation to propose a new multiuser detection framework, within the context of CDMA systems, to combat multiple access interference in fastfading multipath channels [13]. Our development is based on the TF RAKE receiver, and includes TF generalizations of the decorrelating [14] and minimum-mean-squared error (MMSE) [15] multiuser receivers. Analytical and simulation results show that the relatively modest Doppler spreads ${ }^{1}(100-200$ $\mathrm{Hz}$ ) encountered in practical fast-fading scenarios can be transformed into significant joint multipath-Doppler diversity gains by the multiuser receivers proposed in this paper.

In the next section, we develop the relevant TF-based channel and signal models for fast-fading CDMA environments. Section III develops our multiuser detection framework based on the TF RAKE receiver. Analytical and simulated results on the performance of the proposed receivers are provided in Section IV. Section V contains some concluding remarks and directions for future research.

\section{TF-BASEd Channel And Signal Models}

In this section, we provide a brief description of the canonical TF decomposition of the WSSUS [9], [10] in the context of

\footnotetext{
${ }^{1}$ Sufficient to degrade the performance of existing systems due to errors in channel state estimation [6], [8].
} 


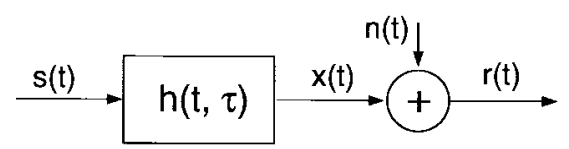

Fig. 1. Mobile wireless channel: a linear time-varying system.

multiuser CDMA systems. The channel representation serves as the backbone of the multiuser detection framework developed in this paper.

\section{A. Single-User Channel Representation}

As depicted in Fig. 1, the complex baseband signal $x(t)$ at the output of the channel is related to the transmitted complex baseband signal $s(t)$ by

$$
x(t)=\int h(t, \tau) s(t-\tau) d \tau
$$

where $h(t, \tau)$ is the time-varying impulse response of the channel [1]. An equivalent representation is in terms of the channel spreading function $H(\theta, \tau)$ [1], [16]

$$
\begin{aligned}
x(t) & =\int_{0}^{T_{m}} \int_{-B_{d}}^{B_{d}} H(\theta, \tau) s(t-\tau) e^{j 2 \pi \theta t} d \theta d \tau \\
H(\theta, \tau) & \stackrel{\text { def }}{=} \int h(t, \tau) e^{-j 2 \pi \theta t} d t .
\end{aligned}
$$

The channel produces time and frequency shifts in the signal and the output signal $x(t)$ is a linear combination of TFshifted copies of $s(t)$. In (2), $T_{m}$ is the multipath spread of the channel and denotes the maximum delay produced by the channel. Similarly, $B_{d}$ is the Doppler spread and denotes the maximum (one-sided) Doppler shift introduced by the channel. Fast-fading channels encountered in practice exhibit Doppler spreads on the order of $100-200 \mathrm{~Hz}$ due to relative motions of the users [6], [8].

The dynamics of the channel are best described statistically, and the WSSUS model assumes that $H(\theta, \tau)$ is a two-dimensional uncorrelated Gaussian process [1], [16]

$$
\mathrm{E}\left[H(\theta, \tau) H^{*}\left(\theta^{\prime}, \tau^{\prime}\right)\right]=\Psi(\theta, \tau) \delta\left(\theta-\theta^{\prime}\right) \delta\left(\tau-\tau^{\prime}\right)
$$

where $\delta(x)$ denotes the Dirac delta function. The function $\Psi(\theta, \tau) \geq 0$ is called the scattering function [1], [16]. The multipath spread $T_{m}$ is the maximum (essential) support of $\Psi(\theta, \tau)$ in the $\tau$ direction, and the Doppler spread $B_{d}$ is its maximum (one-sided) support in the $\theta$ direction.

For a spread-spectrum signal $s(t)$ of duration $T$ and chip interval $T_{c}$, the WSSUS channel admits the following canonical finite-dimensional decomposition [9]-[11]:

$$
x(t) \approx \frac{T_{c}}{T} \sum_{l=0}^{L} \sum_{m=-M}^{M} H^{m l} s\left(t-l T_{c}\right) e^{j \frac{2 \pi m t}{T}}, \quad 0 \leq t<T
$$

with $L=\left\lceil T_{m} / T_{c}\right\rceil, M=\left\lceil T B_{d}\right\rceil$ and

$$
H^{m l} \stackrel{\text { def }}{=} \hat{H}\left(m / T, l T_{c}\right)
$$

where $\hat{H}(\theta, \tau)$ is a TF-smoothed version of $H(\theta, \tau)$

$$
\begin{aligned}
\hat{H}(\theta, \tau)= & \frac{T}{T_{c}} \int_{0}^{T_{m}} \int_{-B_{d}}^{B_{d}} H\left(\theta^{\prime}, \tau^{\prime}\right) e^{-j \pi\left(\theta-\theta^{\prime}\right) T} \\
& \times \operatorname{sinc}\left(\left(\theta-\theta^{\prime}\right) T\right) \operatorname{sinc}\left(\left(\tau-\tau^{\prime}\right) / T_{c}\right) d \theta^{\prime} d \tau^{\prime} .
\end{aligned}
$$

The channel samples $H^{m l}$ are approximately uncorrelated

$$
E\left[H^{m l} H^{* m^{\prime} l^{\prime}}\right] \approx \hat{\Psi}\left(m / T, l T_{c}\right) \delta_{m-m^{\prime}} \delta_{l-l^{\prime}}
$$

where $\hat{\Psi}(\theta, \tau)=E\left[|\hat{H}(\theta, \tau)|^{2}\right]$ and the waveforms $u_{m l}(t) \stackrel{\text { def }}{=}$ $s\left(t-l T_{c}\right) e^{j 2 \pi m t / T}$ are approximately orthogonal [9], [10]

$$
\int u_{m l}(t) u_{m^{\prime} l^{\prime}}^{*}(t) d t \approx \delta_{l-l^{\prime}} \delta_{m-m^{\prime}} \int|s(t)|^{2} d t
$$

where $\delta_{k}$ denotes the Kronecker delta function.

The representation (5) is a Karhunen-Loève-like expansion of the received signal in terms of the uncorrelated random variables $H^{m l}$ 's, and the orthogonal waveforms $u_{m l}(t)$ 's. In (5), $L$ denotes the number of multipath components and $M$ the number of Doppler components that are contributing significant energy to the channel [10]. ${ }^{2}$ We note that similar sampled representations for the WSSUS channel have been developed in [16] based on time and bandwidth constraints. However, our formulation and interpretation in terms of diversity signaling is quite different.

The power of the channel representation (5) comes from the fact that it facilitates full exploitation of the inherent channel diversity. The $(L+1)(2 M+1)$ uncorrelated channel samples $H^{m l}$, corresponding to the TF shifted signal copies $s\left(t-l T_{c}\right) e^{j 2 \pi m t / T}$, serve as independent fading channels to provide a substantially higher level of diversity as compared to conventional systems. ${ }^{3}$ In particular, resolving the first $(M=1)$ Doppler components yields $3(L+1)$-level joint multipath-Doppler diversity, which is three times higher than the $(L+1)$-level multipath diversity attained by existing systems. As we will see, the TF RAKE receiver provides the mechanism for exploiting joint multipath-Doppler diversity by computing the correlator outputs corresponding to the waveforms $s\left(t-l T_{c}\right) e^{j 2 \pi m t / T}$.

We note that values of $T B_{d} \approx 0.01-0.015$, produced by commonly encountered Doppler spreads, significantly degrade the performance of the RAKE receiver due to errors in channel state estimation [6], [8]. More importantly, at modestly larger (0.2-0.6) values of $T B_{d}$, significant multipath-Doppler diversity gains can be achieved via appropriate signal processing [10], [17]. There are several approaches for exploiting multipath-Doppler diversity by attaining larger values of $T B_{d}$ [9]-[11], [17] (more discussion in Section III-C).

\section{B. Multiuser Signal Model}

For a CDMA system with $K$ users and employing synchronous coherent binary phase-shift keying (BPSK) signaling, such as may be encountered in the downlink of a mobile communication system [2], [18], the signal at the input of the receiver is given by

$$
r(t)=x(t)+n(t)=\sum_{i=-I}^{I} \sum_{k=1}^{K} b_{k}(i) x_{k}^{i}(t)+n(t)
$$

\footnotetext{
${ }^{2}$ The approximation in (5) can be made arbitrarily close by including more summation terms and sufficiently finer sampling in multipath. However, most of the energy is captured by the $(L+1)(2 M+1)$ terms in (5) [10].

${ }^{3} \mathrm{An}$ arbitrarily high level of diversity can be achieved by decreasing $T_{C}$ (increasing bandwidth; finer multipath resolution) and/or increasing $T$ (finer Doppler resolution). Exploiting Doppler diversity corresponds to the latter. Note that the two resolutions are not constrained by the uncertainty principle.
} 
where $b_{k}(i) \in\{-1,1\}$ is the $i$ th bit of the $k$ th user, $x_{k}^{i}(t)$ is the unmodulated received baseband signal for the $i$ th bit of the $k$ th user, $I$ is the size of the detection window, and $n(t)$ is the complex baseband additive white Gaussian noise (AWGN) with power spectral density $\mathcal{N}_{0}$. In terms of the representation (5), the signal $x_{k}^{i}(t)$ can be expressed as

$$
x_{k}^{i}(t) \approx \frac{T_{c}}{T} \sum_{l=0}^{L} \sum_{m=-M}^{M} H_{k}^{m l}(i) s_{k}\left(t-i T-l T_{c}\right) e^{j \frac{2 \pi m t}{T}}
$$

where $s_{k}(t)$ is the spreading waveform of the $k$ th user and $H_{k}^{m l}(i)$ are the channel coefficients corresponding to the $i$ th bit of the $k$ th user. ${ }^{4}$ Note that we have absorbed the signal powers and the carrier phases for the different users in the channel coefficients $H_{k}^{m l}(i) .^{5}$

For simplicity of exposition, we introduce a vector notation for the signals. Let us start by defining

$$
s_{k}^{m l}(t) \stackrel{\text { def }}{=} s_{k}\left(t-l T_{c}\right) e^{j \frac{2 \pi m t}{T}}
$$

and let $\mathbf{s}(t)$ denote the $K(L+1)(2 M+1) \times 1$ vector

$$
\mathbf{s}(t) \stackrel{\text { def }}{=}\left[\mathbf{s}_{1}^{T}(t), \mathbf{s}_{2}^{T}(t), \ldots, \mathbf{s}_{K}^{T}(t)\right]^{T}
$$

where the $(L+1)(2 M+1) \times 1$ vectors $\mathbf{s}_{k}(t)$ are given by

$$
\begin{aligned}
\mathbf{s}_{k}(t) \stackrel{\text { def }}{=} & {\left[\mathbf{s}_{k}^{-M^{T}}(t), \mathbf{s}_{k}^{-M+1^{T}}(t), \ldots, \mathbf{s}_{k}^{0^{T}}(t), \ldots,\right.} \\
& \left.\mathbf{s}_{k}^{M-1^{T}}(t), \mathbf{s}_{k}^{M^{T}}(t)\right]^{T}, \quad k=1,2, \ldots, K
\end{aligned}
$$

in terms of the $(L+1) \times 1$ vectors

$$
\begin{aligned}
\mathbf{s}_{k}^{m}(t) & \stackrel{\text { def }}{=}\left[s_{k}^{m 0}(t), s_{k}^{m 1}(t), \ldots, s_{k}^{m L}(t)\right]^{T} \\
m & =-M,-M+1, \ldots, 0, \ldots, M-1, M .
\end{aligned}
$$

Similarly, as with $\mathbf{s}_{k}(t)$ and $\mathbf{s}_{k}^{m}(t)$, define the $(L+1)(2 M+$ 1) $\times 1$ vectors $\mathbf{h}_{k}(i)$ in terms of the $(L+1) \times 1$ vectors $\mathbf{h}_{k}^{m}(i)$, which are in turn defined in terms of $H_{k}^{m l}(i)$. Finally, define the $K(L+1)(2 M+1) \times K$ channel matrix for the $i$ th symbol as

$$
\mathbf{H}(i) \stackrel{\text { def }}{=}\left[\begin{array}{cccc}
\mathbf{h}_{1}(i) & \mathbf{0} & \cdots & \mathbf{0} \\
\mathbf{0} & \mathbf{h}_{2}(i) & \mathbf{0} & \cdots \\
\vdots & \vdots & \ddots & \vdots \\
\mathbf{0} & \cdots & \mathbf{0} & \mathbf{h}_{K}(i)
\end{array}\right]
$$

and the $K \times 1$ vector for the $i$ th bits as

$$
\mathbf{b}(i) \stackrel{\text { def }}{=}\left[b_{1}(i), b_{2}(i), \ldots, b_{K}(i)\right]^{T} .
$$

In terms of the above notation, the received signal $r(t)$ can be expressed as

$$
r(t) \approx \sum_{i=-I}^{I} \mathbf{s}^{T}(t-i T) \mathbf{H}(i) \mathbf{b}(i)+n(t) .
$$

Thus, the received signal is a linear combination of the TFshifted signals $s_{k}^{m l}(t)$, which also define the front-end TF

\footnotetext{
${ }^{4}$ For simplicity of notation, we use the same $L$ and $M$ for all the users. Our discussion, however, can be extended straightforwardly to incorporate different values of $L$ and $M$ for different users.

${ }^{5}$ Note that for the downlink (mobile-to-user) the receiver sees identical channels for the different users; $H_{k}^{m l}=H^{m l}$ for all $k$. However, in the uplink (user-to-mobile) the channels are typically distinct.
}

correlators (TF RAKE receiver) for realizing the sufficient statistics for detecting the bits of different users.

For negligible intersymbol interference (ISI) $\left(T_{m} \ll T\right)$, the output of the TF correlators for the $p$ th bit is given by the $K(L+1)(2 M+1) \times 1$ vector

$$
\mathbf{z}(p) \stackrel{\text { def }}{=} \int r(t) \mathbf{s}^{*}(t-p T) d t \approx \mathbf{R H}(p) \mathbf{b}(p)+\mathbf{w}
$$

where

$$
\mathbf{R} \stackrel{\text { def }}{=} \int \mathbf{s}^{*}(t) \mathbf{s}^{T}(t) d t=\left[\begin{array}{cccc}
\mathbf{R}_{11} & \mathbf{R}_{12} & \cdots & \mathbf{R}_{1 K} \\
\mathbf{R}_{21} & \mathbf{R}_{22} & \cdots & \mathbf{R}_{2 K} \\
\vdots & \vdots & \vdots & \vdots \\
\mathbf{R}_{K 1} & \mathbf{R}_{K 2} & \cdots & \mathbf{R}_{K K}
\end{array}\right]
$$

$$
\mathbf{R}_{k k^{\prime}} \stackrel{\text { def }}{=} \int \mathbf{s}_{k}^{*}(t) \mathbf{s}_{k^{\prime}}^{T}(t) d t
$$

and

$$
\mathrm{w} \stackrel{\text { def }}{=} \int \mathrm{s}^{*}(t-p T) n(t) d t
$$

is a zero-mean complex Gaussian noise vector with $E\left[\mathrm{ww}^{H}\right]=\mathcal{N}_{0} \mathbf{R}$. It follows that in the absence of ISI, the one-shot detector suffices in which the decision about the $p$ th bit is based on the received waveform for the corresponding bit only. Thus, in subsequent sections we suppress the bit index $p$ and, without loss of generality, focus on the 0th bit: $\mathbf{z}=\mathbf{R H b}+\mathbf{w}$.

Recall that the $k$ th component of $\mathbf{z}=\left[\mathbf{z}_{1}, \mathbf{z}_{2}, \ldots, \mathbf{z}_{K}\right]^{T}$ consists of the TF correlator outputs for the $k$ th user

$$
z_{k}^{m l}=\int r(t) s_{k}^{* m l}(t) d t=\int r(t) s_{k}^{*}\left(t-l T_{c}\right) e^{-j 2 \pi m t / T} d t .
$$

In fact, the front-end TF correlators are a generalization of the RAKE receiver and can be efficiently implemented via a bank of RAKE receivers [9]-[11]. This is the reason for referring to the proposed receiver structures for joint multipath-Doppler processing as TF RAKE receivers.

\section{Multiuser Detectors for Fast-Fading Channels}

In this section we develop a multiuser detection framework that incorporates the fundamental multipath-Doppler channel model of Section II to deliver near-far resistant receiver structures for fast-fading channels. The TF RAKE receiver plays a central role in our development, and we start our discussion with the computationally intensive optimal multiuser detector which is an extension of the synchronous receiver derived in [19]. The structure of the optimal detector inspires a unified formulation of a class of suboptimal, near-far resistant receiver structures that are computationally tractable. The resulting multiuser TF RAKE receivers include generalizations of the decorrelating [14] and MMSE [15] multiuser detectors. Restricted to slow-fading scenarios $[M=0$ in (5)], our treatment also serves as a unified formulation of the multiuser RAKE receivers proposed in [4], [5], and [20]. 


\section{A. Minimum Probability of Error Receiver}

Recall the definition of $\mathbf{z}=\left[\mathbf{z}_{1}, \mathbf{z}_{2}, \ldots, \mathbf{z}_{K}\right]$ in (19). In the absence of multiple access interference, corresponding to the single-user case, the optimal receiver for each user is the TF RAKE receiver with maximal-ratio-combining (MRC) [9], [10]

$$
\begin{aligned}
\hat{b}_{k} & =\operatorname{sign}\left\{\operatorname{Re}\left[\mathbf{h}_{k}^{H} \mathbf{z}_{k}\right]\right\} \\
& =\operatorname{sign}\left\{\operatorname{Re}\left[\sum_{l=0}^{L} \sum_{m=-M}^{M} H_{k}^{* m l} z_{k}^{m l}\right]\right\}, \quad k=1,2, \ldots, K
\end{aligned}
$$

which coherently combines the different multipath-Dopplershifted signal components to achieve $(L+1)(2 M+1)$-order diversity. ${ }^{6}$ Note that MRC requires the knowledge of the channel coefficients $H_{k}^{m l}$ which, for example, may be estimated through a pilot transmission. ${ }^{7}$ The signal component of $\mathbf{z}_{k}$ in this case is $\mathcal{E}_{k} \mathbf{h}_{k} b_{k}$ where $\mathcal{E}_{k} \stackrel{\text { def }}{=}\left\|s_{k}\right\|^{2}=\int\left|s_{k}(t)\right|^{2} d t$, resulting in $\mathcal{E}_{k}\left\|\mathbf{h}_{k}\right\|^{2} b_{k}$ as the real-valued signal component of the test statistic in (24). Of course, in the presence of other users the detector in (24) is not near-far resistant since it ignores multiple access interference.

It is instructive to study the structure of the optimal multiuser detector which essentially augments the single-user receiver (24) by suppressing multiple access interference. Following the approach in [19], it can be shown that the minimum probability of error reception is achieved by the maximum likelihood (ML) receiver given by

$$
\begin{aligned}
\hat{\mathbf{b}}_{\text {opt }} & =\arg \max _{\mathbf{b} \in\{-1,1\}^{K}} p(\mathbf{z} \mid \mathbf{b}) \\
& =\arg \max _{\mathbf{b}}-(\mathbf{z}-\mathbf{R H b})^{H} \mathbf{R}^{-1}(\mathbf{z}-\mathbf{R H b}) \\
& =\arg \max _{\mathbf{b}}\left[2 \operatorname{Re}\left[\mathbf{b}^{T} \mathbf{H}^{H} \mathbf{z}\right]-\mathbf{b}^{T} \mathbf{H}^{H} \mathbf{R H b}\right]
\end{aligned}
$$

where the last equality is equivalent to

$$
\begin{aligned}
\hat{\mathbf{b}}_{\text {opt }}=\arg \max _{\mathbf{b}} & {\left[\sum_{k=1}^{K} 2 \operatorname{Re}\left[\mathbf{h}_{k}^{H} \mathbf{z}_{k}\right] b_{k}\right.} \\
& \left.-\sum_{k=1}^{K} \sum_{k^{\prime}=1}^{K} b_{k}\left[\mathbf{H}_{k}^{H} \mathbf{R H}_{k^{\prime}}\right] b_{k^{\prime}}\right]
\end{aligned}
$$

where $\mathbf{H}_{\mathbf{k}}$ is the $k$ th column of $\mathbf{H}$. Note that the effects of fast fading are incorporated in the above formulation via the multipath-Doppler channel coefficient matrix $\mathbf{H}$. The first term corresponds to the single-user TF RAKE receiver and the second term eliminates the multiple access interference. Given the knowledge of channel coefficients, the optimal decision requires a search over $2^{K}$ possibilities for $\mathbf{b}$, which can be efficiently executed with Viterbi sequence decoding [19]. Due to the exponential computational complexity (in the number of users) of the optimal receiver, however, lower complexity,

\footnotetext{
${ }^{6}$ The conventional single-user RAKE receiver corresponds to $M=0$ in (24).

${ }^{7}$ Reliable estimation of $H_{k}^{m l}$ is affected by the rate of temporal variations of $H_{k}^{m l}$. We note that noncoherent techniques, which rely less on channel estimation, are also possible in our framework [21]. Though important, channel estimation issues are beyond the scope of this paper.
}

suboptimal approaches are sought in practice. Next, we discuss an approach for designing suboptimal near-far-resistant TF RAKE receivers that are computationally tractable.

\section{B. A Class of Suboptimal Near-Far-Resistant Receivers}

The structure of the suboptimal receivers that we derive is motivated by the optimal single-user MRC detector (24). As evident from (24), the MRC detector makes the decision by coherently combining the TF correlator outputs $\mathbf{z}_{k}$ corresponding to each user. As mentioned before, in the absence of other users, the signal component of $\mathbf{z}_{k}$ is

$$
\left.\int r(t) \mathbf{s}_{k}^{*}(t) d t\right|_{r(t)=x(t)}=\mathcal{E}_{k} \mathbf{h}_{k} b_{k} .
$$

In our suboptimal approach, the basic idea is to obtain an estimate of the noise-free correlator outputs, $\mathbf{h}_{k} b_{k}$ and then to coherently combine them as in (24) to obtain the bit estimates for each user. The estimation procedure for $\mathrm{h}_{k} b_{k}$ should be such that the resulting detector is near-far resistant. In terms of the $K$ users, we seek a near-far resistant estimate of

$$
\mathbf{y}=\left.\int r(t) \mathbf{s}^{*}(t) d t\right|_{r(t)=x(t)}=\mathbf{H b} \text {. }
$$

The nature of the estimate of $y$ determines the structure of the receivers. For computational efficiency both the receiver structures that we propose employ a linear estimate. Generically, the estimate of $\mathbf{y}$ takes the form

$$
\hat{\mathbf{y}}=\mathbf{F z}=\mathbf{F R H b}+\mathbf{F w}=\mathbf{y}_{s}+\mathbf{y}_{n}
$$

where $\mathbf{y}_{n} \sim \mathcal{N}(\mathbf{0}, \mathbf{Q})$ with

$$
\begin{aligned}
\mathbf{Q} & =E\left[\mathbf{F w w}^{H} \mathbf{F}^{H}\right]=\mathcal{N}_{0} \mathbf{F R F} \mathbf{F}^{H} \\
& =\left[\begin{array}{cccc}
\mathbf{Q}_{11} & \mathbf{Q}_{12} & \cdots & \mathbf{Q}_{1 K} \\
\mathbf{Q}_{21} & \mathbf{Q}_{22} & \cdots & \mathbf{Q}_{2 K} \\
\vdots & \vdots & \vdots & \vdots \\
\mathbf{Q}_{K 1} & \mathbf{Q}_{K 2} & \cdots & \mathbf{Q}_{K K}
\end{array}\right] .
\end{aligned}
$$

The matrix $\mathbf{F}$ is chosen to yield a near-far resistant estimate of $\mathbf{y}$. Following the application of $\mathbf{F}$, MRC is applied to the different multipath-Doppler components of each user $\left(\hat{\mathbf{y}}_{k}\right)$, analogous to (24). Since the noise in the estimate $\hat{\mathbf{y}}$ is correlated, however, a prewhitening operation is needed. The general form of the overall multiuser TF RAKE receiver becomes

$$
\hat{\mathbf{b}}=\operatorname{sign}\left\{\operatorname{Re}\left[\mathbf{H}^{H} \mathrm{D} \hat{\mathbf{y}}\right]\right\}=\operatorname{sign}\left\{\operatorname{Re}\left[\mathbf{H}^{H} \mathbf{D F z}\right]\right\}
$$

where the block-diagonal matrix D

$$
\mathbf{D}=\left[\begin{array}{cccc}
\mathbf{Q}_{11}^{-1} & \mathbf{0} & \cdots & \mathbf{0} \\
\mathbf{0} & \mathbf{Q}_{22}^{-1} & \mathbf{0} & \cdots \\
\vdots & \vdots & \ddots & \vdots \\
\mathbf{0} & \cdots & \mathbf{0} & \mathbf{Q}_{K K}^{-1}
\end{array}\right]
$$

performs the prewhitening and the matrix $\mathbf{H}^{H}$ performs MRC. A schematic of the overall receiver structure is depicted in Fig. 2. In the following subsections we discuss two special cases of the receiver structure (31), based on the choice of the estimator matrix $\mathbf{F}$. 


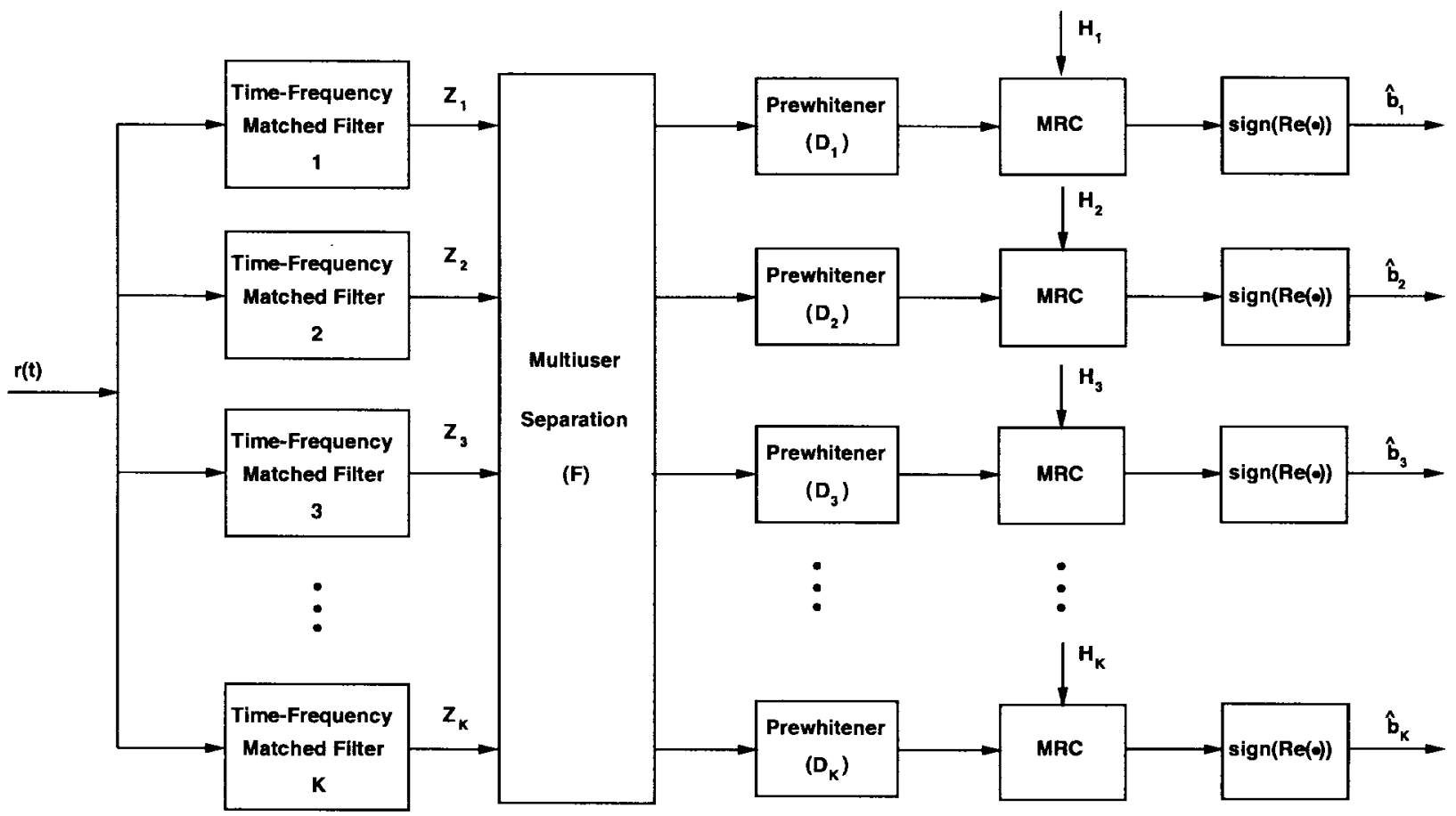

Fig. 2. Generic structure of the proposed multiuser TF RAKE receivers.

1) ML Estimation-Decorrelating Receiver: If the ML estimate of $\mathbf{y}=\mathbf{H b}$ is employed, the resulting receiver is linear and constitutes a generalization of the decorrelating receiver [14], [4]. The estimator matrix $\mathbf{F}$ in this case is

$$
\mathbf{F}_{\mathrm{ML}}=\mathbf{R}^{-1}
$$

since

$$
\hat{\mathbf{y}}_{\mathrm{ML}}=\arg \max _{\mathbf{y}}\left[2 \operatorname{Re}\left[\mathbf{z}^{H} \mathbf{y}\right]-\mathbf{y}^{H} \mathbf{R y}\right]=\mathbf{R}^{-1} \mathbf{z} .
$$

Note that the correlation matrix of the transformed noise vector is $\mathbf{Q}_{\mathrm{ML}}=\mathcal{N}_{0} \mathbf{R}^{-1}$. From (31) it follows that the resulting decorrelating TF RAKE receiver takes the form

$$
\begin{aligned}
\hat{\mathbf{b}}_{\mathrm{dec}} & =\operatorname{sign}\left\{\operatorname{Re}\left[\mathbf{H}^{H} \mathbf{D}_{\mathrm{dec}} \hat{\mathbf{y}}_{\mathrm{ML}}\right]\right\} \\
& =\operatorname{sign}\left\{\operatorname{Re}\left[\mathbf{H}^{H} \mathbf{D}_{\mathrm{dec}} \mathbf{R}^{-1} \mathbf{z}\right]\right\}
\end{aligned}
$$

where $\mathbf{D}_{\text {dec }}$ is defined as in (32), with $\mathbf{Q}=\mathbf{Q}_{\mathrm{ML}}$. The decorrelating TF RAKE receiver in (35) combats fast multipath fading by exploiting joint multipath-Doppler diversity, and attains near-far resistance via the ML estimate in (34).

2) MMSE Receiver: If a linear MMSE estimate of $\mathbf{y}=\mathbf{H b}$ is employed, a generalization of the MMSE detector proposed in [15], [5] is obtained. In this case, the estimator matrix $\mathbf{F}$ solves

$$
\mathbf{F}_{\text {mmse }}=\arg \min _{\mathbf{F}} E\|\mathbf{H b}-\mathbf{F} \mathbf{z}\|^{2}
$$

and, as shown in the Appendix, it takes the form

$$
\mathbf{F}_{\text {mmse }}=\left(\mathbf{R}+\mathcal{N}_{0} \Psi^{-1}\right)^{-1}
$$

where

$$
\Psi \stackrel{\text { def }}{=} E\left[\mathbf{H H}^{H}\right] .
$$

The resulting MMSE TF RAKE receiver is given by

$$
\hat{\mathbf{b}}_{\text {mmse }}=\operatorname{sign}\left\{\operatorname{Re}\left[\mathbf{H}^{H} \mathbf{D}_{\text {mmse }} \mathbf{F}_{\text {mmse }} \mathbf{z}\right]\right\}
$$

where $\mathbf{D}_{\text {mmse }}$ is defined as in (32) and the transformed noise correlation matrix is

$$
\mathbf{Q}_{\text {mmse }}=\mathcal{N}_{0}\left(\mathbf{R}+\mathcal{N}_{0} \Psi^{-1}\right)^{-1} \mathbf{R}\left(\mathbf{R}+\mathcal{N}_{0} \Psi^{-1}\right)^{-1} .
$$

Note that $\boldsymbol{\Psi}$ is a function of the powers $p_{k}$ of different users, and the second-order channel statistics of the corresponding users. Specifically, for the WSSUS channel model $\boldsymbol{\Psi}$ has the following diagonal structure

$$
\boldsymbol{\Psi} \stackrel{\text { def }}{=} E\left[\mathbf{H H}^{H}\right]=\left[\begin{array}{cccc}
p_{1} \boldsymbol{\Psi}_{1} & \mathbf{0} & \cdots & \mathbf{0} \\
\mathbf{0} & p_{2} \boldsymbol{\Psi}_{2} & \cdots & \mathbf{0} \\
\vdots & \vdots & \vdots & \vdots \\
\mathbf{0} & \mathbf{0} & \cdots & p_{K} \boldsymbol{\Psi}_{K}
\end{array}\right]
$$

where $\boldsymbol{\Psi}_{k}$ is a diagonal matrix [due to (8)], corresponding to the powers in the different multipath-Doppler channel coefficients $H_{k}^{m l}$ of the $k$ th user. ${ }^{8}$

\section{Discussion}

The proposed framework for multiuser detection over fastfading channels promises improved performance on two counts: 1) the underlying TF RAKE receiver is optimally matched to the fast-fading WSSUS channel and 2) the resulting

${ }^{8}$ Note that for the downlink the channel statistics are identical for all the users; $\Psi_{k}=\Psi$. For the uplink, in general, the $\boldsymbol{\Psi}_{k}$ are distinct. 
systems achieve an inherently higher level of diversity due to joint multipath-Doppler processing.

As mentioned before, even the relatively small Doppler spreads encountered in practice can be leveraged into significant diversity gains via appropriate signal processing [10], [17]. This is due to the fact that a large fraction of the maximum diversity gain due to additional (Doppler) diversity components is attained at relatively small fractions $(5 \%-10 \%)$ of total power in those components [10], [17]. Such performance gains are inherited by the multiuser receivers proposed in this paper, as demonstrated in the next section.

Several system modalities that can benefit from joint multipath-Doppler diversity are identified in [9]-[11]. One particularly promising technique is the use of time-selective signaling and reception to achieve sufficiently large $(\geq 0.2)$ values of $T B_{d}$ [17], [11]. The basic idea is to use signaling waveforms that are longer than the intersymbol period, thereby introducing overlap between successive symbol waveforms. For example, $T B_{d} \approx 0.013$ for a data rate of $10 \mathrm{kHz}$, carrier frequency of $1.8 \mathrm{GHz}$, and maximum vehicle speed of 50 $\mathrm{mi} / \mathrm{h}$. Spreading codes that are about 16 times longer than the conventional symbol yield $T B_{d} \approx 0.2$, facilitating significant joint multipath-Doppler diversity gains (3-6 dB) [17]. Clearly, the overlap between successive symbols introduces ISI. Initial studies, however, in the single-user case indicate that the excellent autocorrelation properties of pseudorandom codes result in virtually negligible ISI [17]. Integrating time-selective signaling into the multiuser formulation developed in this paper warrants further research. We note, however, that the interference-suppression-based formulation adopted in this paper will automatically account for the additional multiple access ISI introduced by time-selective signaling.

Finally, we note that the concept of exploiting Doppler diversity in a multiuser CDMA framework based on timeselective signaling is similar in spirit to the ideas proposed in [22] and [23]. In fact, the concept of Doppler diversity can be integrated into the formulation in [22] and [23] to provide an alternative interpretation of the results. For a more detailed comparison of the two approaches, we refer the reader to [17].

\section{PeRformance Analysis}

The main objective of this section is to quantify the joint multipath-Doppler diversity gains attainable by the multiuser TF RAKE receivers in practical fast-fading scenarios. To provide a reference, we compare the performance of the proposed receivers under realistic fast fading, with that of the conventional multiuser RAKE receiver operating under ideal slow fading conditions. Our results demonstrate that even the relatively small Doppler spreads encountered in practice can yield significant gains via joint multipath-Doppler processing.

Of the two multiuser detectors proposed in the last section, only the decorrelating receiver lends itself to tractable performance analysis. Proceeding in a manner analogous to that of the standard performance analysis for the decorrelating detector [14], [4], we first derive an expression for the probability of bit error for the decorrelating receiver, and then provide simulated results for both the decorrelating and MMSE TF RAKE receivers.

Recall from (35) that the decorrelating receiver is of the form

$$
\hat{\mathbf{b}}=\operatorname{sign}\{\operatorname{Re}[\mathbf{g}]\}
$$

where the test statistic $\mathbf{g}$ is given by

$$
\mathbf{g}=\mathbf{H}^{H} \mathbf{D}_{\mathrm{dec}} \mathbf{H b}+\mathbf{H}^{H} \mathbf{D}_{\mathrm{dec}} \mathbf{R}^{-1} \mathbf{w} .
$$

It follows that the test statistic $g_{k}$ corresponding to the $k$ th user is

$$
g_{k}=\mathbf{h}_{k}^{H} \mathbf{Q}_{k k}^{-1} \mathbf{h}_{k} b_{k}+\mathbf{h}_{k}^{H} \mathbf{Q}_{k k}^{-1} \tilde{\mathbf{w}}_{k}=g_{s, k}+g_{n, k}
$$

where $\tilde{\mathbf{w}}_{k}$ is the component of the vector $\mathbf{R}^{-1} \mathbf{w}$ corresponding to the $k$ th user. It can be readily verified that $E\left[\left|g_{n, k}\right|^{2} \mid \mathbf{h}_{k}\right]=$ $\mathbf{h}_{k}^{H} \mathbf{Q}_{k k}^{-1} \mathbf{h}_{k}$. Thus, the probability of bit error for the $k$ th user, conditioned on the knowledge of the channel coefficients $\mathbf{h}_{k}$, is [1]

$$
P_{k \mid \mathbf{h}_{k}}=Q\left(\sqrt{2 \mathbf{h}_{k}^{H} \mathbf{Q}_{k k}^{-1} \mathbf{h}_{k}}\right)
$$

where $Q(x)=\frac{1}{\sqrt{2 \pi}} \int_{x}^{\infty} e^{-x^{2} / 2} d x$. Note that since $\mathbf{h}_{k}$ consists of independent complex Gaussian random variables

$$
\mathbf{h}_{k}^{H} \mathbf{Q}_{k k}^{-1} \mathbf{h}_{k}=\sum_{l=1}^{(L+1)(2 M+1)} \lambda_{l} \gamma_{l}
$$

where the $\lambda_{l}$ 's are the eigenvalues of $p_{k} \mathbf{Q}_{k k}^{-1} \boldsymbol{\Psi}_{k}$ and the $\gamma_{l}$ 's are independent $\chi^{2}$ random variables each with two degrees of freedom, and $E\left[\left|\gamma_{l}\right|^{2}\right]=1$. The unconditional probability of bit error can be obtained by averaging $P_{k \mid \mathbf{h}_{k}}$ with respect to the probability density function of $\mathbf{h}_{k}^{H} \mathbf{Q}_{k k}^{-1} \mathbf{h}_{k}$ and is given by $[1, \mathrm{pp} .801-802]$

$$
P_{k}=\sum_{l=1}^{(L+1)(2 M+1)} \frac{\pi_{l}}{2}\left[1-\sqrt{\frac{\lambda_{l}}{1+\lambda_{l}}}\right]
$$

where

$$
\pi_{l}=\prod_{i=1, i \neq l}^{(L+1)(2 M+1)} \frac{\lambda_{l}}{\lambda_{l}-\lambda_{i}} .
$$

Note that the eigenvalues $\lambda_{i}$ 's include the dependence on the signal powers $p_{k}$ and $\mathcal{N}_{0}$.

All the numerical results presented next are based on coherent BPSK signaling with perfect channel knowledge, and employ $M$-sequences of length $N=T / T_{c}=63$ as the spreading codes. Moreover, in slow fading, we assume that two-level $(L=1)$ multipath diversity is achievable with a uniform multipath power profile. For simulating practical fastfading scenarios we assume that in addition to the multipath components the $m=\mp 1$, Doppler components contribute about $10 \%$ of the total power in the channel, corresponding to six-level joint multipath-Doppler diversity with a $(0.05$, $0.9,0.05)$ Doppler power profile for $m=-1,0,1$. As noted earlier, such a Doppler profile may be achieved in practice by employing appropriate signal processing, such as timeselective signaling and reception [10], [11], [17]. 


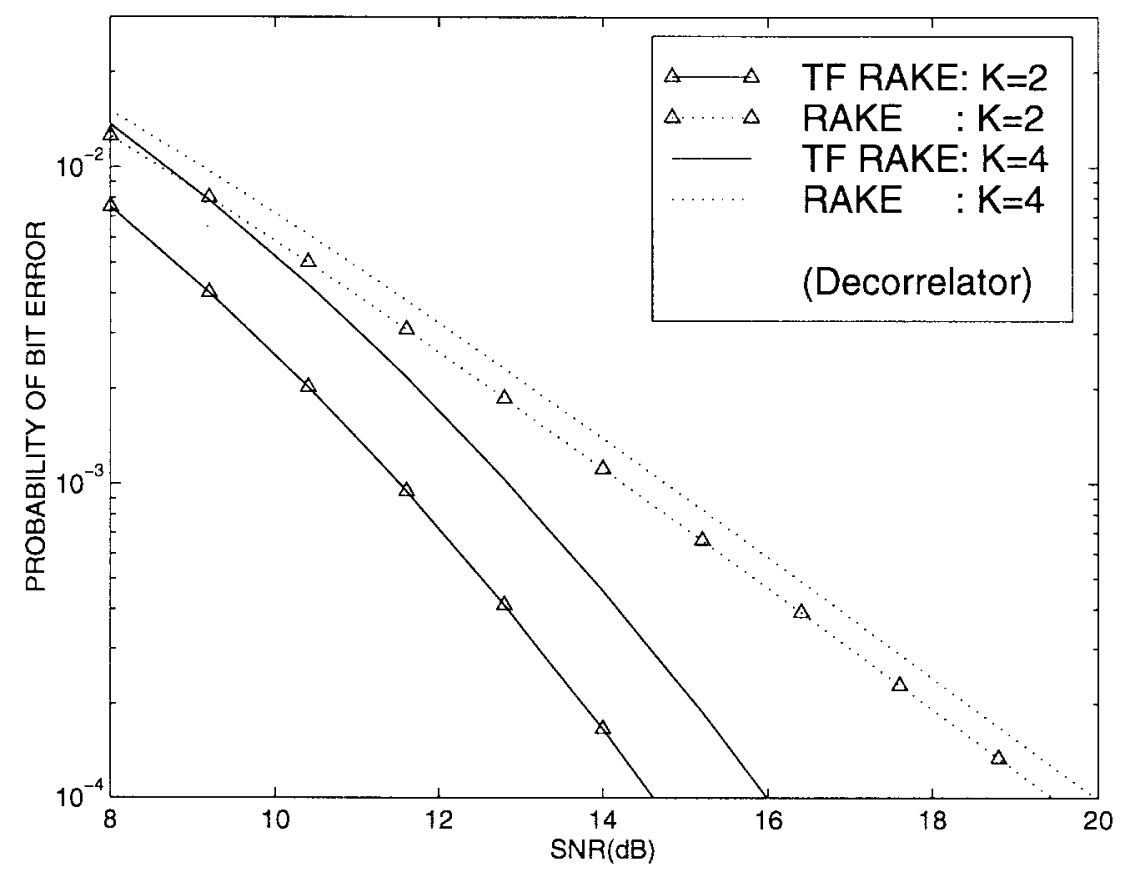

Fig. 3. Comparison between the analytical performance of the TF and conventional decorrelating RAKE receivers for two and four users.

\section{A. Numerical Results: Decorrelating TF RAKE Receiver}

First, we calculate the analytical probability of bit error $\left(P_{e}\right)$ based on (47) for the decorrelating TF RAKE receiver and the conventional decorrelating RAKE receiver $(M=0)$, as a function of signal-to-noise ratio (SNR). Fig. 3 shows the results for $K=2$ and 4 users. Evidently, by exploiting joint multipath-Doppler diversity corresponding to only $10 \%$ of the total power in the two Doppler components, the multiuser TF RAKE receiver significantly outperforms the conventional multiuser RAKE receiver. For example, for $K=2$ users, the TF receiver achieves a 5-dB SNR gain at $P_{e}=10^{-4}$. Moreover, as expected, as the number of users increases, a higher SNR is needed to achieve a prescribed value of $P_{e}$, due to increased interference. As illustrated in Fig. 4, however, the loss in performance for higher number of users is reduced for longer spreading codes due to improved correlation properties (and, hence, reduced interference). Moreover, the performance gains of the TF RAKE receiver increase monotonically with increasing SNR (decreasing $P_{e}$ ).

Fig. 5 shows the performance results based on the Monte Carlo simulation. Evidently, analytical and simulated results agree closely. Analytical results are slightly optimistic for $K=4$ users.

\section{B. Numerical Results: MMSE TF RAKE Receiver}

Fig. 6 compares the simulated performance of the multiuser MMSE receivers. The results are similar to those obtained for the decorrelating receiver, demonstrating superior performance of the TF RAKE receiver due to joint multipath-Doppler diversity.

Fig. 7 demonstrates the near-far resistance of the proposed MMSE TF RAKE receiver by plotting the $P_{e}$ for the first (desired) user (at SNR $=14 \mathrm{~dB}$ ) as a function of the SNR of the second user, relative to that of the first user. Based on similar performance of the two multiuser receivers, we expect similar behavior for the decorrelating receiver.

Finally, Fig. 8 illustrates the effect of the choice of spreading codes on the performance of the proposed receivers. Comparison is made between $M$-sequences, which have wellknown characteristics, with randomly generated sequences. The results, based on the MMSE receivers, indicate that the choice of spreading codes has no significant effect on performance.

\section{CONCLUSION}

Multipath fading and multiple access interference are two of the most significant factors limiting the performance of existing mobile wireless CDMA systems. Receiver structures that combine multiuser detection and multipath diversity processing have been developed recently to address the two problems. However, the performance of existing multiuser RAKE receivers degrades significantly in fast fading encountered in many mobile communication scenarios. In particular, systems based on the conventional RAKE receiver exhibit limiting bit error rates that cannot be improved by increasing the transmitted power. In practice, additional diversity is needed for improved performance.

In this paper, we have proposed a novel framework for multiuser detection that achieves an inherently higher level of diversity via joint multipath-Doppler processing. At the heart of our approach is a TF RAKE receiver, derived from a canonical TF decomposition of the fast-fading channel, that facilitates joint multipath-Doppler diversity. The proposed multiuser TF RAKE receiver structures are near-far resistant and include generalizations of the conventional decorrelating and MMSE receivers. Analytical and simulated results demonstrate that the relatively modest Doppler spreads encountered in practice can be transformed into significant diversity gains by the proposed multiuser systems. 


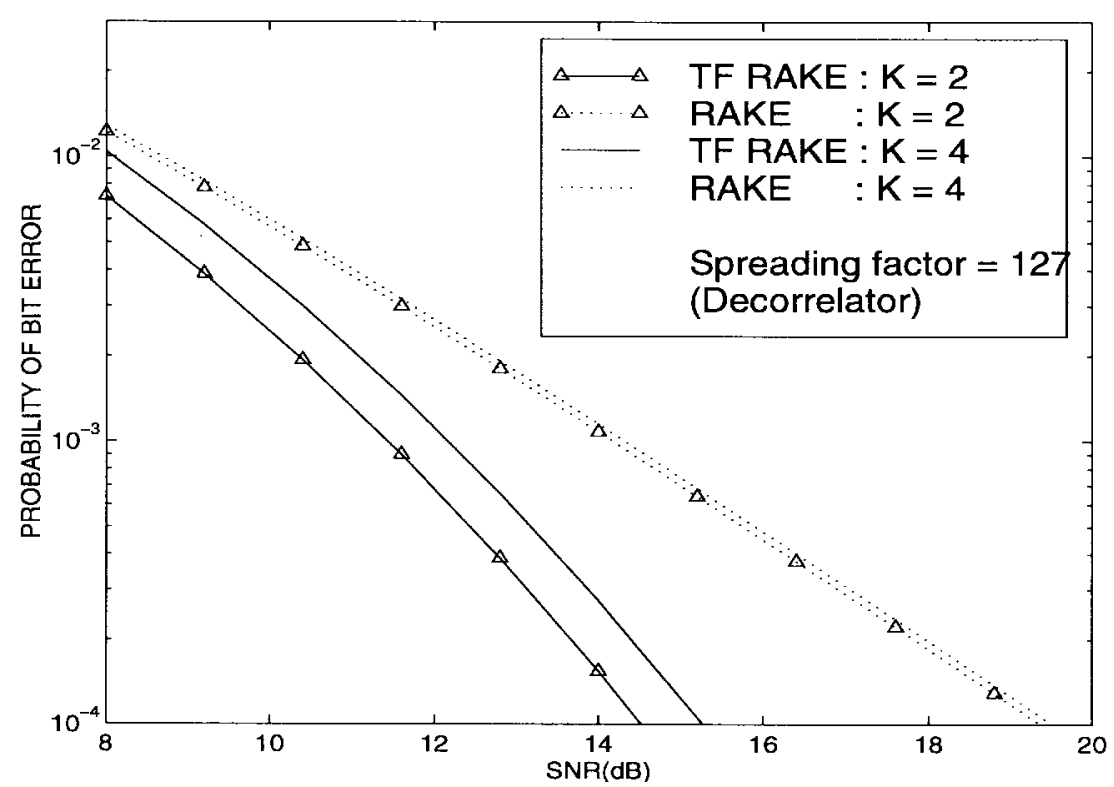

(a)

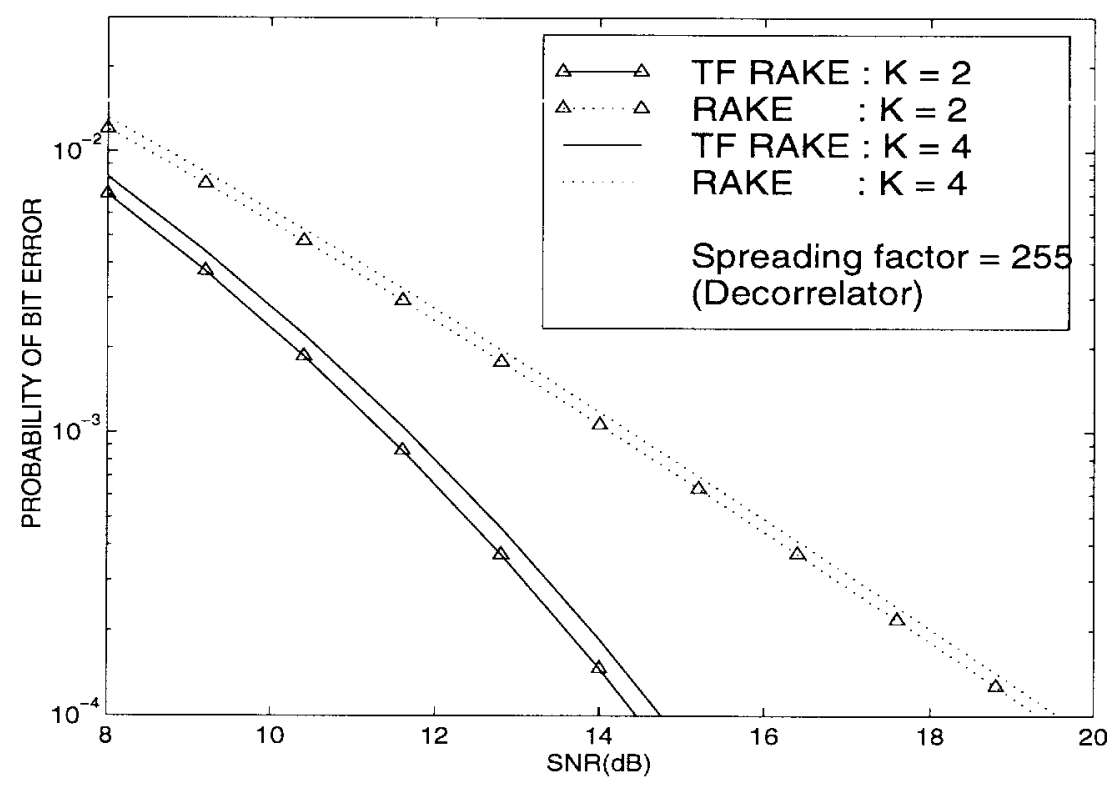

(b)

Fig. 4. Analytical performance of the decorrelating RAKE receivers for longer spreading codes: (a) $N=127$ and (b) $N=255$. The difference in performance for two and four users is smaller for longer codes.

The multiuser TF RAKE receivers proposed in this paper espouse the paradigm of first removing multiple access interference, followed by MRC of the multipath-Doppler components. However, receiver structures that employ diversity combining first are also possible. According to a study of multiuser RAKE receivers [24], first employing diversity combining yields better performance with perfect knowledge of channel parameters, whereas executing interference suppression first, as in our approach, may be more robust if channel estimates are used. Investigation of the two approaches in the context of fast fading warrants further investigation.

From a diversity viewpoint, we note that an arbitrarily high level of diversity can be achieved in the proposed systems by employing time-selective signaling and reception based on spreading codes that are substantially longer than the intersymbol duration [10], [11], [17]. For long codes, in addition to improved performance, the system is also less affected by the number of users, as indicated by our analysis. Moreover, such systems effectively transform the fading channel into an AWGN channel (in the limit) [17], thereby facilitating the use of powerful coding techniques developed for the AWGN channel.

Finally, we note that even though we restricted our discussion to the synchronous case, for clarity of exposition, the results of this paper can be readily extended to asynchronous scenarios. Moreover, the multipath-Doppler diversity framework can also be leveraged to develop powerful techniques for noncoherent detection and multiuser timing acquisition [21]. 


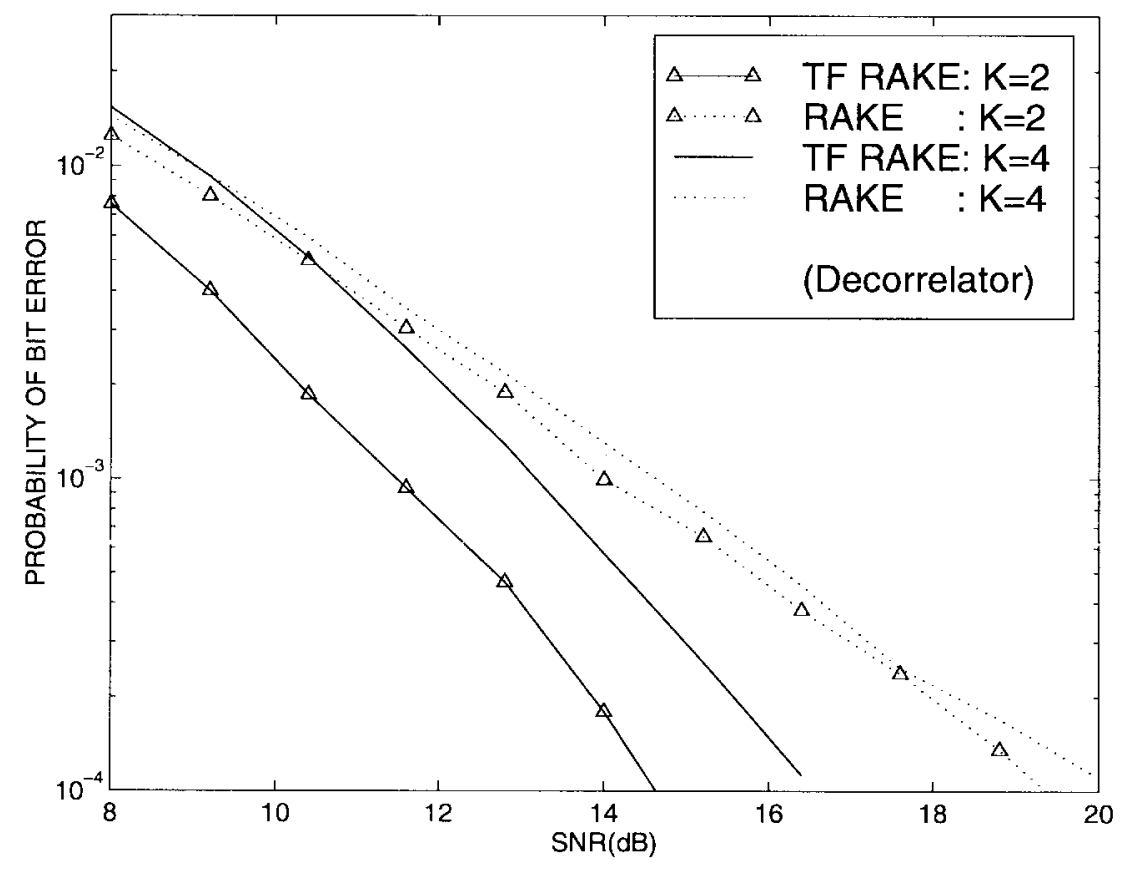

Fig. 5. Comparison between the simulated performance of the TF and conventional decorrelating RAKE receivers for two and four users.

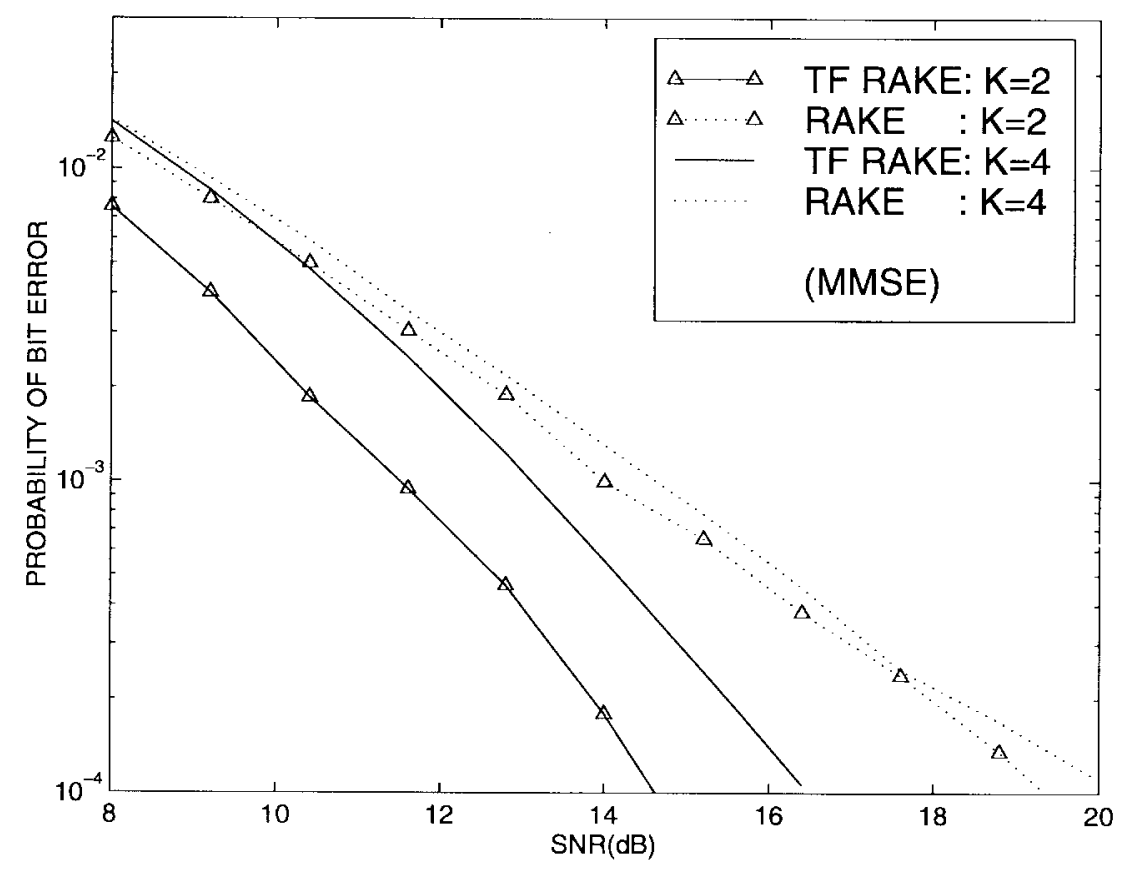

Fig. 6. Comparison between the simulated performance of the TF and conventional MMSE RAKE receivers for two and four users.

\section{APPENDIX}

Recall that

$$
\hat{\mathbf{y}}_{\text {mmse }}=\mathbf{F}_{\text {mmse }} \mathbf{z}
$$

where $\mathbf{F}_{\text {mmse }}$ solves

$$
\mathbf{F}_{\text {mmse }}=\arg \min _{\mathbf{F}} E\|\mathbf{H b}-\mathbf{F} \mathbf{z}\|^{2}
$$

The MSE functional can be expanded as

$$
\begin{aligned}
E \| & \mathbf{H b}-\mathbf{F z} \|^{2} \\
= & E\left[\mathbf{z}^{H} \mathbf{F}^{H} \mathbf{F} \mathbf{z}\right]+E\left[\mathbf{b}^{T} \mathbf{H}^{H} \mathbf{H b}\right]-2 \operatorname{Re}\left\{E\left[\mathbf{z}^{H} \mathbf{F}^{H} \mathbf{H b}\right]\right\} \\
= & \operatorname{Tr}\left[\mathbf{F} E\left[\mathbf{z z}{ }^{H}\right] \mathbf{F}^{H}\right]+\operatorname{Tr}\left[E\left[\mathbf{H}^{H} \mathbf{H}\right]\right] \\
& -2 \operatorname{Re}\left\{\operatorname{Tr}\left[\mathbf{F}^{H} E\left[\mathbf{H} \mathbf{b} \mathbf{z}^{H}\right]\right]\right\} \\
= & \operatorname{Tr}\left[\mathbf{F} \mathbf{Q}_{1} \mathbf{F}^{H}\right]+\operatorname{Tr}\left[E\left[\mathbf{H}^{H} \mathbf{H}\right]\right]-2 \operatorname{Re}\left\{\operatorname{Tr}\left[\mathbf{F}^{H} \mathbf{Q}_{2}\right]\right\}
\end{aligned}
$$




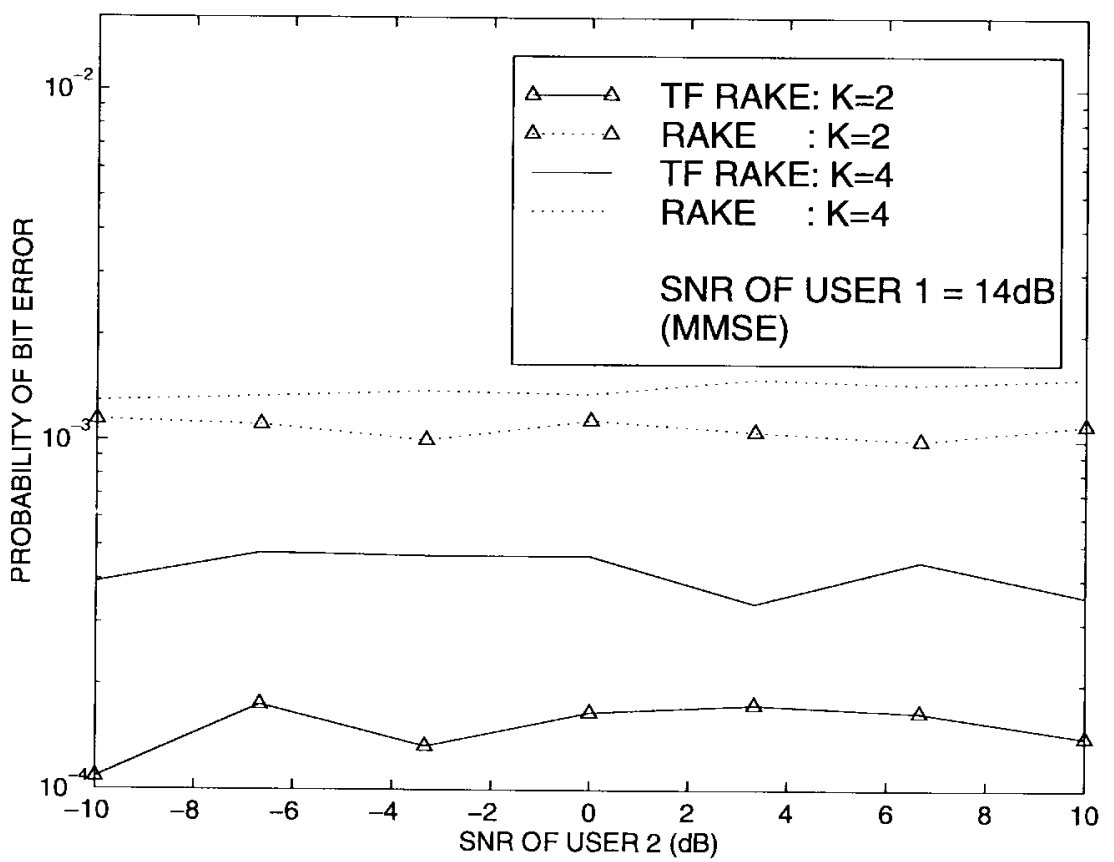

Fig. 7. Near-far resistance of the MMSE RAKE receivers (simulation results).

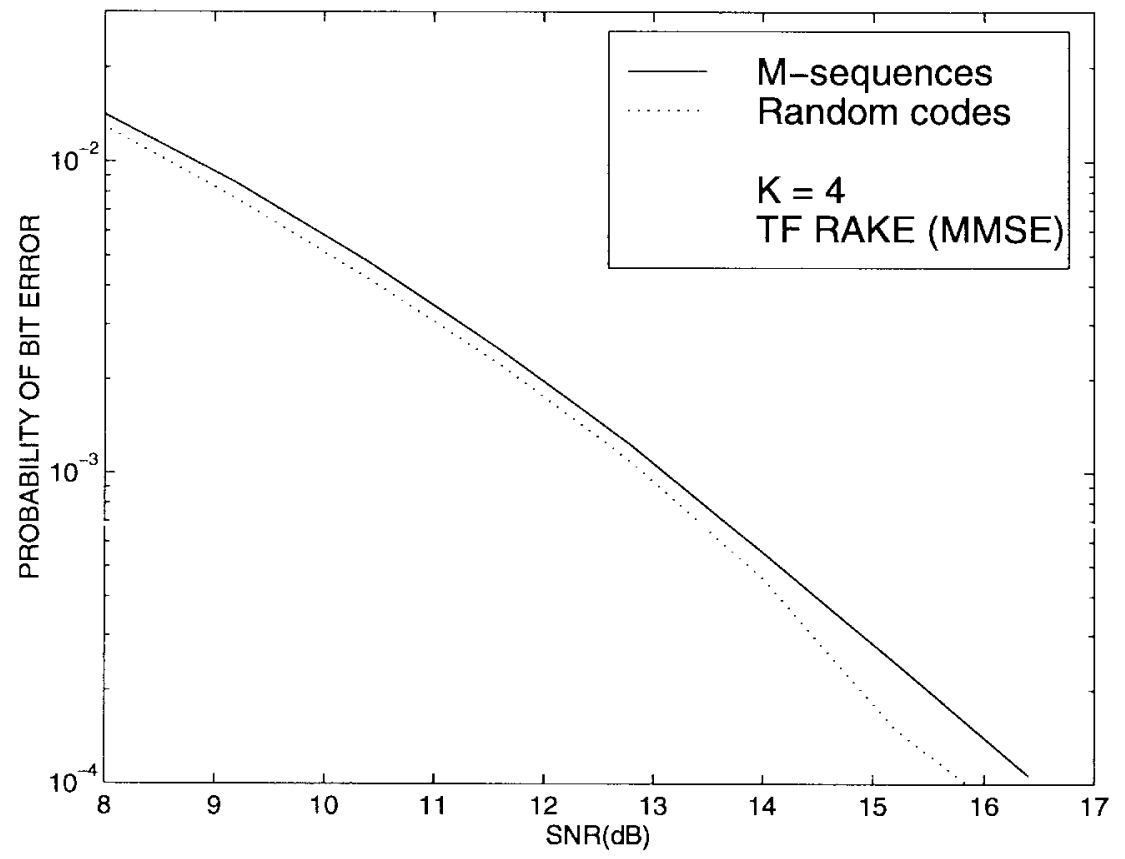

Fig. 8. Effect of the choice of spreading codes on performance of MMSE TF RAKE receiver.

where $\operatorname{Tr}[\cdot]$ denotes the trace of a matrix. Using $\mathbf{z}=\mathbf{R H b}+$ w we get

$$
\mathbf{Q}_{1} \stackrel{\text { def }}{=} E\left[\mathbf{z z}^{H}\right]=\mathbf{R} \Psi \mathbf{R}+\mathcal{N}_{0} \mathbf{R}
$$

where $\boldsymbol{\Psi}=E\left[\mathbf{H H}^{H}\right]$ and we have used the fact that $E\left[\mathrm{bb}^{T}\right]=\mathbf{I}$. Similarly,

$$
\mathbf{Q}_{2} \stackrel{\text { def }}{=} E\left[\mathbf{H b z}^{H}\right]=\mathbf{\Psi} \mathbf{R} \text {. }
$$

Differentiating $E\|\mathbf{H b}-\mathbf{F} \mathbf{z}\|^{2}$ in (51) with respect to $\mathbf{F}$, we have

$$
\frac{\partial E\|\mathbf{H b}-\mathbf{F} \mathbf{z}\|^{2}}{\partial \mathbf{F}}=2 \mathbf{Q}_{1} \mathbf{F}^{H}-2 \mathbf{Q}_{2}^{H}
$$

where we have used the following identities:

$$
\begin{aligned}
\frac{\partial \operatorname{Tr}\left[\mathbf{F} \mathbf{Q F}{ }^{H}\right]}{\partial \mathbf{F}} & =2 \mathbf{Q F}^{H} \\
\frac{\partial \operatorname{Re}\left\{\operatorname{Tr}\left[\mathbf{F}^{H} \mathbf{Q}\right]\right\}}{\partial \mathbf{F}} & =\mathbf{Q}^{H} .
\end{aligned}
$$

Setting the gradient in (54) equal to zero yields the optimal $\mathbf{F}$

$$
\mathbf{F}_{\text {mmse }}=\mathbf{Q}_{2} \mathbf{Q}_{1}^{-1}=\left(\mathbf{R}+\mathcal{N}_{0} \Psi^{-1}\right)^{-1}
$$

where we have used (52) and (53). 


\section{ACKNOWLEDGMENT}

The authors would like to thank the reviewers for constructive comments. In particular, they thank one of the reviewers for pointing out the connection of the channel representation (5) to similar developments in [16].

\section{REFERENCES}

[1] J. G. Proakis, Digital Communications, 3rd ed. New York: McGrawHill, 1995.

[2] T. S. Rappaport, Wireless Communications. Englewood Cliffs, NJ: Prentice-Hall, 1996.

[3] S. Moshavi, "Multi-user detection for DS-CDMA communications," IEEE Commun. Mag., pp. 124-136, Oct. 1996.

[4] Z. Zvonar and D. Brady, "Linear multipath-decorrelating receivers for CDMA frequency-selective fading channels," IEEE Trans. Commun., vol. 44, pp. 650-653, June 1996.

[5] M. Latva-aho and M. Juntti, "Modified adaptive LMMSE receiver for DS-CDMA systems in fading channels," in Proc. PIMRC'97, Helsinki, Finland, 1997, pp. 554-558.

[6] J. K. Cavers, "An analysis of pilot symbol assisted modulation for Rayleigh fading channels," IEEE Trans. Veh. Technol., vol. 40, pp. 689-693, Nov. 1991.

[7] H. Andoh, M. Sawahashi, and F. Adachi, "Channel estimation using time multiplexed pilot symbols for coherent Rake combining for DS-CDMA mobile radio," in Proc. PIMRC'97, 1997, pp. 954-958.

[8] B. Sklar, "Rayleigh fading channels in mobile digital communication system part II: Mitigation,” IEEE Commun. Mag., pp. 103-109, July 1997.

[9] A. M. Sayeed and B. Aazhang, "Exploiting Doppler diversity in mobile wireless communications," in Proc. 1997 Conf. Information Science Systems (CISS'97), Baltimore, MD, 1997, pp. 287-292.

[10] A. M. Sayeed and B. Aazhang, "Joint multipath-Doppler diversity in mobile wireless communications," IEEE Trans. Commun., to be published.

[11] A. M. Sayeed and B. Aazhang, "Communication over multipath fading channels: A time-frequency perspective," in Wireless Communications: TDMA Versus CDMA, S. G. Glisic and P. A. Lippänen Eds. Norwell, MA: Kluwer, 1997, pp. 73-98.

[12] F. Hlawatsch and G. F. Boudreaux-Bartels, "Linear and quadratic timefrequency signal representations," IEEE Signal Processing Mag., Apr 1992.

[13] A. M. Sayeed, A. Sendonaris, and B. Aazhang, "Multiuser detectors for fast-fading multipath channels," in Proc. 31st Asilomar Conf. Signals, Systems and Computers, Pacific Grove, CA, Nov. 1997, pp. 603-608.

[14] R. Lupas and S. Verdu, "Near-far resistance of multiuser detectors in asynchronous channels," IEEE Trans. Commun., vol. 38, pp. 496-508, Apr. 1990.

[15] U. Madhow and M. L. Honig, "MMSE interference suppression for direct-sequence spread-spectrum CDMA," IEEE Trans. Commun., vol. 42, pp. 3178-3188, Dec. 1994.

[16] P. A. Bello, "Characterization of randomly time-variant linear channels," IEEE Trans. Commun. Syst., vol. CS-11, pp. 360-393, Nov. 1963.

[17] S. Bhasyam, A. M. Sayeed, and B. Aazhang, "Time-selective signaling and reception for communication over multipath fading channels," IEEE Trans. Commun., to be publlished.

[18] A. J. Viterbi, CDMA: Principles of Spread Spectrum Communications. Reading, MA: Addison Wesley, 1995

[19] S. Verdu, "Minimum probability of error for asynchronous Gaussian multiple-access channels," IEEE Trans. Inform. Theory, vol. IT-32, pp. 85-96, Jan. 1986

[20] Z. Zvonar, "Combined multiuser detection and diversity reception for wireless CDMA systems," IEEE Trans. Veh. Technol., vol. 45, pp. 205-211, Feb. 1996.

[21] A. M. Sayeed and B. Aazhang, "Multiuser timing acquisition over multipath fading channels," in Proc. 1998 Conf. Information Science Systems (CISS'98), 1998.

[22] G. W. Wornell, "Spread-signature CDMA: Efficient multiuser communications in the presence of fading," IEEE Trans. Inform. Theory, vol. 41, pp. 1418-1438, Sept. 1995.

[23] G. W. Wornell, "Spread-response precoding for communication over fading channels," IEEE Trans. Inform. Theory, vol. 42, pp. 488-501, Mar. 1996.
[24] H. C. Huang, S. C. Schwartz, and S. Verdu, "Combined multipath and spatial resolution for multiuser detection: Potentials and problems," in Proc. 1995 Int. Symp. Information Theory, 1995, p. 380.

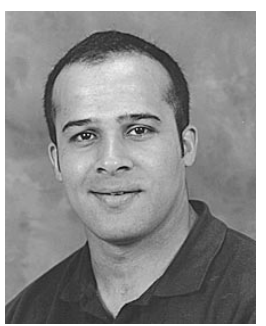

Akbar M. Sayeed (S'89-M'97) received the B.S degree in electrical engineering from the University of Wisconsin, Madison, in 1991 and the M.S. and Ph.D. degrees in electrical engineering from the University of Illinois at Urbana-Champaign in 1993 and 1996, respectively.

While at the University of Illinois, he was a Research Assistant at the Coordinated Science Laboratory and a Schlumberger Fellow in signal processing. During 1996-1997, he was a postdoctoral fellow at Rice University, Houston, TX. Since August 1997 he has been with the University of Wisconsin, Madison, where he is currently an Assistant Professor of Electrical and Computer Engineering. His research interests are in signal processing for wireless communications, statistical and time-varying signal processing, and time frequency and wavelet analysis.

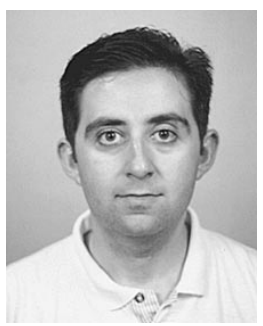

Andrew Sendonaris (S'93) received the B.S. (summa cum laude) and M.S. degrees in electrical engineering from Rice University, Houston, TX, in 1993 and 1995, respectively.He is currently working toward the Ph.D. degree at Rice University.

His research interests include multiple access communications over fading multipath channels, code division multiple access (CDMA) systems, the capacity of and resource allocation for cellular mobile radio networks, and digital transmission over twisted-pair copper loops.

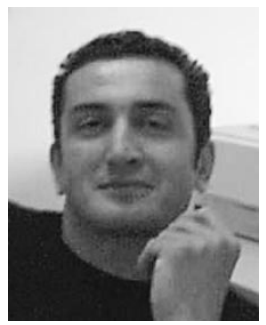

Behnaam Aazhang (S'81-M'85-SM'91) received the B.S. (highest hons.), M.S., and Ph.D. degrees in electrical and computer engineering from the University of Illinois at Urbana-Champaign in 1981, 1983 , and 1986, respectively.

From 1981 to 1985 , he was a Research Assistant in the Coordinated Science Laboratory at the University of Illinois. In August 1985 he joined the faculty of Rice University, Houston, TX, where he is now a Professor in the Department of Electrical and Computer Engineering and the Director of the Center for Multimedia Communications. He has been a Visiting Professor at IBM Federal Systems Company, Houston, TX, the Laboratory for Communication Technology at the Swiss Federal Institute of Technology (ETH), Zurich, Switzerland, the Telecommunications Laboratory at University of Oulu, Oulu, Finland, and at the U.S. Air Force Phillips Laboratory, Albuquerque, NM. His research interests are in the areas of communication and information theory and their applications, with emphasis on multiple access communications; cellular mobile radio communications; optical communication networks; and efficient simulation of stochastic systems.

Dr. Aazhang is a recipient of the 1993 Alcoa Foundation Award, the 1987-1989 NSF Engineering Initiation Award, and the 1984-1985 IBM Graduate Fellowship. He is a member of Tau Beta Pi and Eta Kappa Nu. $\mathrm{He}$ is currently serving as the Editor for spread spectrum networks for the IEEE TRAnsactions on Communications, the Treasurer of the IEEE Information Theory Society, and the Technical Area Chair of the 1997 Asilomar Conference, Pacific Grove, CA. From 1990-1993 he served as the Secretary of the Information Theory Society and was the Publications Chairman of the 1993 IEEE International Symposium on Information Theory, San Antonio, TX. 